\title{
CATEGORIAL GRAMMARS FOR STRATA OF NON-CF LANGUAGES AND THEIR PARSERS
}

\author{
Micha1 P. Chytil \\ Charles University \\ Malostranské nám. 25 \\ 11800 Praha 1 \\ Czeohoslovakia
}

\section{Abstract}

We introduce a goneralization of categorial grammar extending its desoriptive power, and a simple model of oategorial Erammar parser. Both tools can be adjusted to partioular strata of languages via restricting grammatical or computationa.1 complexity.

\section{Two questions about categoria1 grammars}

In. spite of the fascinating fornal simplicity and lucidity of categorial. granmax as developed by Bar-HilleI [1], Lambek [7] and followers, it has nevertheless never been brought into wide scale use. Why is this so?

We may easily" recopnize two drawbacks.

1 Restricted scope of oategorial grammars.

It was shown early $[1]$ that the set of Languages describable by these grammars is exactly that of context-free languages. Is this restriction inevitable or can a similar type of language description be retained beyond the limit of contextm free Langtages? This is the first question we try to answer.

2/ No realistic model of categorial Errammar parsing.

The schematic description of catogorial analysis of a given sentenoe $a_{1} \ldots$ ... $a_{n}$ is sketched in Fi.g. 1.

assign a category $c_{i}$ to each sentence nember $a_{i}$

cancel the string of categories to the target category $t$

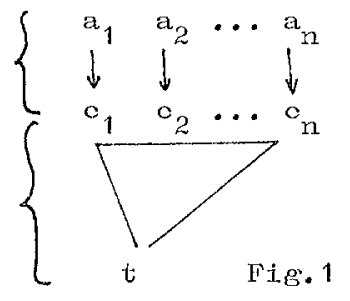

Hans Karlgren

ICVAL Södermalmstorg 8 11645 Stookholm Sweden.

This abstract soheme cannot serve as a description of a realistio parsing procedure. The suitable assigmement appearing here as the first phase is in fact the goal of the parsing. The "brute force" approach following the above scheme, which cheoks all possible assignements and tries to cancel them i.s not computationa11y traotable, since for most grammars the number of all possible assigmements grows exponentially with the length of the analysed sentence.

The moral of this observation is that the assignement cannot be separated from the cancellation. Similarly as parsers based on phrase - structure grammaxs have to make at each point of time an inteliigent chotce of rule to apply noxt, the categorial parser mut make an intelligent choice out of a Iist of altomative categories. This necessjty to look ahoad at cancellation when making the assignement leads to the conolusion [6] that assigmement and canoellation must in any actual parser be interwoven. Therefore our second key question reads:

Can this interweaving be grasped by a simple formal model or does it unavoidingily lead to a mess of complicated ad hoo and heuristic techni.ques?

II. Proposed solution

We introduce in nontechnica.1 language the essence of the proposed generalization of categorial grammars and their parsers. The exact mathematioal formulations can be found in [3].

Grammars. The principal difference betwoen the "classical" categorial grammar and the generalized categorial grammar (GCG) is 
that instead of finite sets of categories corresponding to texminal symbols, GCG allows for infinite sets of categories. Each such infinite set, however, can be generated by a simple procedure, in fact a procedure based on a finite state generator.

Automata. Wo offor list automaton (LA) as a mathematical model of categorial grammar parsing. List automaton is schematically represented by rig. 2.

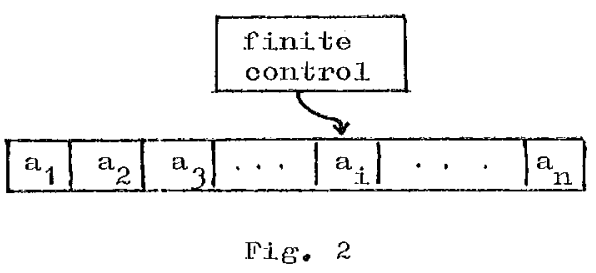

LA consigts of a nondeterministic rinite state control unit at taohed to a finite tape. At the beginning of the computation, the tape contains the analysed string. The automaton can read and rewrite scanned. symbols and move the scanning head one tape coll to the left or right analogously as Turing machine. In addition to $i t$, it can delete the scanned celi, i.e. cat it out and paste the remaining tape parts together.

In the remainder of the paragraph we li.st rosults indicating, as wo bolieve, that the conoepts of GCG and LA give satisfactory answexs to the above questions. a) Soope and mutual correspondence. Both GCGs and LA represent exact $1 y$ a11 context; -sensitive languages. Similarly liko in the case of Cli-granmans and pushdown automata or context-sensitive grammars and linearly bounded automata [5] there exist transfomations of GCGs to LA and vice versa: an algoxithm $\Lambda_{1}$, which for each GCG G yielas a IAA $A_{1}(G)$ representing the same language and conversoly an algorithm $\Lambda_{2}$ which for each $L \Lambda$ M yields an equivalent $\mathrm{GCG} \mathrm{A}_{2}(\mathrm{M})$.

The next step in our argument is to point out a remarkable feature of the interplay botweon GCGs and IA.

b/ Stratification. The correspondence betwoen GCGs and LA can be observed not only in the whole olass of context-sensitive languages, but also on the level of CF-languagos and in each of infinitely many strata between. CP a CS-languages. The stratification can be derined vi.a two complexity measures.

Grammatical complexity: given a $\mathrm{GCC}$ C and a string $w$, the grammatical oomplexity of $w$ wrt. $G$, denoted $\mathrm{G}(\mathrm{w})$, is defined as the length of the longest oam tegory used in the analysis wrt. o . (For ambiguous grammars, the complexity is defined for each parse of the string). Computational complexity: given a IA $M$ and a string $w$, the computational complexity of $w$ wrt., denoted $M(w)$, is defined as the maximal number of visits paid to a single square during the acoepting computation (ambiguity being treated as bo:(ore).

In the light of these complexity measures we can reconsicter the relation between GCGs and LA determined by the above montioned algorithms $\Lambda_{1}$ and $\Lambda_{2}$ For any GCG $G$ and any sentence $w$, each graumatical description of $w$ wrt. $G$ is reflected as a computation of $\Lambda_{1}(G)$ accepting $w$. The granmatical complexity of the description is approximately the same as the computational complexity of the corresponding computation. Analogous result holds for $\Lambda_{2}$.

Now, any Punction $f$ mapping natural numbers on natural numbers determines a stratum $S(f)$ of langrtages: a language $L$ belongs to the stratum $S(S)$ iff and only if it can be represented by a GCG G (or equivalently a LA M) such that from each sertence $w$ from $L$ of length $\mathrm{n}$, the complexity $G(w)$ (or $M(w)$ ) does not exceed the number $f(n)$. Our previous considerations show that the a.Igorithms $A_{1}, A_{2}$ respect the stratifi-.. cation. Ilence the introduced tools can be 
adjusted to the investigated languages.

Two exanples:

1/ The grammars in the stratum $S$ (const)

(detarmined by constant functions) are exaotly Bar-Hillel categonial grammars. "Finite visit" LA appear as their parsers. 2/ The Languages in the strata $S(\mathbf{f})$, whore $f$ is any function of order smallex then the function $\log (\log n)$ bolong to "almost context-free languages" (or. [2]) sharing crucial properties of CF-languages.

c/ Assignement and cancellation interwoven. To show that 1.ist automata, besides their simplicity, meet also the above formulated requirement for natural parsers of categorial grammar, we have to examine at least informally in nore detail the rolationship between a GCG $G$ and its parser $\Lambda_{1}(G)$. When the autiomation $A_{1}(G)$ analyses a string $a_{1} \ldots a_{n}$, thon during the m-th visit to a square containing originally a symbol $a_{i}$, the automaton fixes the $m-t h$ symbol in the oategory belonging to $a_{i}$. Thus arter nt visits, 11 symbols of the category are determined. Thercfore from the (tinfinite) set of categonies assignable to to $a_{i}$, only those whi.ch a rree with the determined symbols remain in play. To determine the next symbol of a category, the automaton can check the environment of the square and take into acoount possible cancellations. $\Lambda$ t the moment, when all. symbols in a category" are fixed, the corresponding square is deleted. In other words, a computation of $A_{1}(G)$ on a strine $a_{1} \ldots a_{n}$ evolves dynamtcally a suitable assignement $c_{1} \ldots c_{n}$ of categories. The infomation used by the parser consists of

- generating nechantsm of categories corresponding to partioular symbols, - indioations of possible cancelling with nei.ghbour categories.

The computation is completed at the moment when the assignement is found.
I.I. Open questions

$1 /$ Tn this brief note we tried to grasp what foatures of the exact mathematical models described in [3] we consider to be fundamental. We can imagine altemative models differing in technical details but having the same features. Which of the models should bo chosen as "canonical" wi1l require nore extensive gtudies.

2/ Our considerations deal with nondem teministio LA, i.o. in faot with "methods" of parsing. "Whe step from "nethods" to "a.tgoxithms" leads from nondeterninjstio to deterministic LA. Tren a glimpse of the basic stratum $S$ (const) promises in.teresting resultis an observation of l. Hibbard [4] shows that deterministic "finite visit" la represent a class of Ianguages broader thar. the class of deterninistic context-free languages. It inplies that deteministic categorial. gramnar (i.n the classical. sense) parsing wi.l go beyond the limits of $\odot \cdot g$.

LR-parsing based on Climegranmars.

References

[1] Y. Bar-flitLeL, C.Gaifman, F. Shanits: On categorial and phrase struoturo grammars, Bull. Res.Coundil Israel, F9, 1960

[2] M.P.Chytil: Almost context-free languages, to appear in Fundamenta linformaticae,1986

[3] M.P.ChytiL, II.Karlgren: Categorial grams mars and list automata for strata of nonCF languages, to appeas in $J$ ovan Benthem, W.Buszkowstki, W. Marciszewski (ed.), Categorial grammar, Jo Benjamins $R_{0} V_{0}$, Ans terdam - Philadelhia

[4] T.libbard: A reneralization of contextfroe determinism, Infomnation and Control $11(1967), 196-238$

[5] J.E.Hoperoft, J.D.ULLman: Formal Langua.w ges and their rolation to automata, Add.Wesley 1969

[6] H.Karlgren: Categorial grammar calculus, Soriptor, Stookholn 1974

[7] J. lambels: On the caloulus of symtactic types, in structure of language and its math. aspects, Proc. 12th Symp. Appl. Math. AMS, Providence 1961 